Pacific Journal of Mathematics

COINCIDENCES AND FIXED POINTS OF MULTIFUNCTION
INTO TREES 


\section{COINCIDENCES AND FIXED POINTS OF MULTIFUNCTIONS INTO TREES}

\section{HELGA SCHIRMER}

The main purpose of this paper is to find conditions on an upper semi-continuous (usc) multifunction $\varphi$ from a compact Hausdorff space $X$ onto a tree $T$ so that it has a coincidence with any multifunction $\psi: X \rightarrow T$ which is either continuous or usc and connected-valued. It is shown that it is sufficient (but not necessary) that $\varphi$ be either open or monotone. This result contains as special cases known conditions for coincidence producing single-valued maps onto trees as well as known fixed point theorems for multifunctions on trees. It is used to obtain a new result on fixed points, namely that any composite of an usc and connected-valued and a continuous multifunction of a tree into itself has a fixed point. All proofs make use of the order-theoretic characterization of trees by L. E. Ward, Jr.

A single-valued map $f: X \rightarrow Y$ from a space $X$ into a space $Y$ is called coincidence producing for all maps $g: X \rightarrow Y$ if $f$ has a coincidence with all maps $g$, i.e., if there exists a point $x \in X$ such that $f(x)=g(x)$. Coincidence producing maps onto trees have been studied in [4] and [5], and it was shown there that a map $f: X \rightarrow T$ from a continuum $X$ onto a tree $T$ is coincidence producing if it is either monotone or open. The main purpose of this paper is to extend this result to multifunctions, but the situation is here more complex.

The known fixed point theorems for multifunctions of trees and similar spaces are mainly of two kinds: they require that either the multifunction is continuous (see, e.g., [3]) or that it is upper semicontinuous (usc) and connected-valued (see, e.g., [2], [5], [8], and [9]). The principal result of this paper, Theorem 4.6. below, applies to both of these cases and shows that an open or monotone usc function onto a tree is coincidence producing both for continuous and for usc, connected-valued functions. The theorem includes the results for single-valued maps mentioned above. As was the case there, the condition that the coincidence producing function be either open or monotone is sufficient but not necessary; a condition which is both necessary and sufficient is not yet known.

We apply the theorems on coincidence producing functions to obtain some results on fixed points. In addition to showing that any function of a tree into itself which is either continuous or usc and connected-valued has a fixed point, they show that any function which 
can be factored into two functions such that one is continuous and the other is usc and connected-valued does also (see Theorem 5.2).

Although the contents of this paper are generalizations of [4] and [5] the method of their proof is quite different. It makes use of the order-theoretic characterization of trees given by Ward [7] and is related to arguments used in the fixed point case by Capel and Strother [2] and Ward [9].

The required properties of trees are collected in $\S 2$, those of multifunctions in $\S 3$. The proofs of the coincidence theorems can be found in $\S 4$, and the applications to fixed points are given in $\S 5$.

2. Properties of trees. By a tree $T$ we mean a continuum (i.e., a compact connected Hausdorff space) in which every pair of distinct points is separated by a third. It is well-known that every tree has a partial order $\leqq$. We define, as usual,

$$
\begin{aligned}
& L(A)=\{y \in T \mid y \leqq x \text { for some } x \in A\}, \\
& M(A)=\{y \in T \mid x \leqq y \text { for some } x \in A\} \text {. }
\end{aligned}
$$

Then Ward [7] has characterized trees as follows.

A compact Hausdorff space $T$ is a tree if and only if it admits a partial order $\leqq$ satisfying

(i) $L(x)$ and $M(x)$ are closed for every $x \in T$,

(ii) if $x<y$ then there exists a $\mathrm{z}$ such that $x<z<y$,

(iii) $L(x) \cap L(y)$ is a nonnull chain (i.e., is linearly ordered) for every $x, y \in T$,

(iv) $M(x) \backslash\{x\}$ is an open set for every $x \in T$.

We define $[x, y]=M(x) \cap L(y)$; then $[x, y]$ is a nonempty chain if $x<y$. A point $m$ is called a maximum of a subset $A$ of $T$ if $m \in A$ and if $m \nless x$ for each $x \in A$. A zero of $A$ is a point $a_{0} \in A$ for which $A \subset M\left(a_{0}\right)$. In the following four lemmas we state some properties of trees which are needed in the proofs of our main results.

LEMMA 2.1. Every nonempty closed subset of $T$ has a maximum.

Proof. See [6, Th. 1].

LEMMA 2.2. Every nonempty closed connected subset of Thas a zero.

Proof. See [2, Lemma 2].

LEMma 2.3. If $A$ is a connected subset of $T \backslash\{y\}$ for some $y \in T$ and if $A \cap M(y) \neq \varnothing$ then $A \subset M(y) \backslash\{y\}$. 
Proof. Otherwise $M(y) \backslash\{y\}$ and $T \backslash M(y)$ would provide a separation of $A$.

LEMma 2.4. If $y \in V$, where $V$ is an open subset of $T$, and if $y<t$ then there exists $a z \in V$ such that $y<z<t$.

Proof. From [2, Lemma 1].

3. Properties of multifunctions. A multifunction $\varphi: X \rightarrow Y$ from a topological space $X$ to a topological space $Y$ is a correspondence which assigns to each point of $X$ at least one point of $Y$. We say that $\varphi: X \rightarrow Y$ is usc (upper semi-continuous) if for every $x \in X, \varphi(x)$ is closed and for each open set $V \subset Y$ with $\varphi(x) \subset V$ there exists an open set $U \subset X$ with $x \in U$ such that $\varphi(U) \subset V$. It is called lsc (lower semi-continuous) if for every $x \in X$ and for every open set $V \subset Y$ with $\varphi(x) \cap V \neq \varnothing$ there exists an open set $U \subset X$ with $x \in U$ such that $\varphi\left(x^{\prime}\right) \cap V \neq \varnothing$ for all $x^{\prime} \in U$. If $\varphi$ is both usc and lsc it is called continuous. We will reserve the term map for continuous single-valued functions.

It is well known that usc of $\varphi: X \rightarrow Y$ implies that $\varphi^{-1}(B)=$ $\{x \in X \mid \varphi(x) \cap B \neq \varnothing\}$ is closed in $X$ for every closed $B \subset Y$, and lsc of $\varphi: X \rightarrow Y$ implies that $\varphi^{-1}(B)$ is open in $X$ for every open $B \subset Y$. If $X$ and $Y$ are compact Hausdorff spaces, then $\varphi: X \rightarrow Y$ is usc if and only if its graph $\{(x, y) \mid x \in X, y \in Y, y \in \varphi(x)\}$ in $X \times Y$ is closed (see [1, p. 112]). Hence in this case the image $\varphi(A)=\cup\{\varphi(x) \mid x \in A\}$ of a closed set $A \subset X$ is closed in $Y$. The image of an open set under a lsc function need not be open, however, even if $X$ and $Y$ are compact Hausdorff.

Our coincidence producing functions will be special kinds of multifunctions. We give their definitions.

Let $\varphi: X \rightarrow Y$ be a multifunction. Then we say that

(i) $\varphi$ is open if $\varphi(A)$ is open for every open $A \subset X$,

(ii) $\varphi$ is connected-valued if $\varphi(x)$ is connected for every $x \in X$,

(iii) $\varphi$ is connected if $\varphi(A)$ is connected for every connected $A \subset X$,

(iv) $\phi$ is monotone if $\phi^{-1}(y)$ is connected for every $y \in Y$.

We shall write $\varphi: X \rightarrow Y$ if $\varphi$ is onto $Y$. The following lemma states some relations between a multifunction $\varphi: X \rightarrow Y$ and its inverse $\varphi^{-1}: Y \rightarrow X$. They are obvious from the definitions and from the remarks about usc functions.

Lemma 3.1. (i) $\varphi: X \rightarrow Y$ is lsc if and only if $\varphi^{-1}: Y \rightarrow X$ is open,

(ii) $\varphi: X \rightarrow Y$ is monotone if and only if $\varphi^{-1}: Y \rightarrow X$ is 
connected-valued,

(iii) if $X$ and $Y$ are compact Hausdorff, then $\varphi: X \rightarrow Y$ is usc if and only if $\varphi^{-1}: Y \rightarrow X$ is usc.

Finally we describe a case where (ii) in Lemma 3.1 can be strengthened to connected instead of connected-valued.

Lemma 3.2. Any use function $\varphi: X \rightarrow T$ from a compact Hausdorff space $X$ onto a tree $T$ is monotone if and only if $\varphi^{-1}: T \rightarrow X$ is usc and connected.

Proof. It is shown in $\left[10, \mathrm{~B}_{4}\right]$ that any usc and connected-valued function from a locally connected Hausdorff space onto a peripherally compact Hausdorff space is connected. (A space $X$ is called peripherally compact if for each $x \in X$ and open set $U$ containing $x$ there exists an open set $V$ with $x \in V \subset U$ which has a compact boundary.) As a tree is locally connected [7, Lemma 4] and as a compact Hausdorff space is clearly peripherally compact, we see that if $\varphi: X \rightarrow T$ is usc and monotone then $\varphi^{-1}: T \rightarrow X$ is not only usc and connected-valued but also connected. The converse is trivial.

4. The coincidence theorems. A coincidence of two multifunctions $\varphi, \psi: X \rightarrow Y$ is a point $x \in X$ such that $\varphi(x) \cap \psi(x) \neq \varnothing$. The purpose of this paragraph is to find conditions for $\varphi$ so that the functions $\varphi, \psi: X \rightarrow T$ from a compact Hausdorff space $X$ into a tree $T$ have a coincidence if $\psi$ is either continuous or usc and connectedvalued.

THEOREM 4.1. If $\varphi: X \rightarrow T$ is usc and open and if $\psi: X \rightarrow T$ is either continuous or usc and connected-valued then $\varphi$ and $\psi$ have a coincidence.

The crux of the proof of the theorem is to consider the set

$$
E=\left\{y \in T \mid M(y) \cap \psi(x) \neq \varnothing \text { for at least one } x \in \varphi^{-1}(y)\right\}
$$

and study its properties if $\varphi$ and $\psi$ have no coincidence. This is done in the next two lemmas. (Note that if $X=T$ and if $\varphi$ is the identity and $\psi=f: X \rightarrow X$ is a map, then $E$ reduces to $\{x \in X \mid x \leqq f(x)\}$, a set that has been used in the proofs of a number of fixed point theorems for functions on ordered spaces.)

LEMma 4.2. If the usc functions $\varphi, \psi: X \rightarrow T$ have no coincidence and if $\varphi: X \rightarrow T$ is onto, then $E$ is closed. 
Proof. We show that the set

$$
T \backslash E=\left\{y \in T \mid M(y) \cap \psi(x)=\varnothing \text { for every } x \in \varphi^{-1}(y)\right\}
$$

is open. Choose a $y_{0} \in T \backslash E$ and take any $x \in \Phi^{-1}\left(y_{0}\right)$. Then $M\left(y_{0}\right) \cap \psi(x)=\varnothing$, and hence

$$
\left\{y_{0}\right\} \times \psi(x) \subset T \times T \backslash G,
$$

where $G=\{(x, y) \in T \times T \mid x \leqq y\}$ denotes the graph of the partial order of $T$. As $G$ is closed in $T \times T$ [6, Lemma 1] and as $\psi(x)$ is compact there exist open sets $V_{1}(x)$ and $V_{2}(x)$ in $T$ such that

$$
\left\{y_{0}\right\} \times \psi(x) \subset V_{1}(x) \times V_{2}(x) \subset T \times T \backslash G,
$$

and as $\psi$ is usc we can find an open set $U(x)$ containing $x$ such that $\psi(U(x)) \subset V_{2}(x)$. The set $\varphi^{-1}\left(y_{0}\right)$ is compact in $X$ as $\varphi$ is usc, hence the open cover $\left\{U(x) \mid x \in \varphi^{-1}\left(y_{0}\right)\right\}$ has a finite subcover

$$
\left\{U\left(x_{i}\right) \mid x_{i} \in \varphi^{-1}\left(y_{0}\right) ; i=1,2, \cdots, n\right\} .
$$

Then $U=\bigcup_{i=1}^{n} U\left(x_{i}\right)$ is open and $\varphi^{-1}\left(y_{0}\right) \subset U$.

As $\varphi: X \rightarrow T$ is usc the function $\varphi^{-1}: T \rightarrow X$ is also usc (see Lemma 3.1, (iii)). Thus there exists for the open set $U \subset X$ with $\varphi^{-1}\left(y_{0}\right) \subset U$ an open set $V$ containing $y_{0}$ such that $\varphi^{-1}(V) \subset U$. Define $V_{0}=V \cap\left[\bigcap_{i=1}^{n} V_{1}\left(x_{j}\right)\right]$. Then $V_{0}$ is open, contains $y_{0}$, and $\phi^{-1}\left(V_{0}\right) \subset U$. We show that $V_{0} \subset T \backslash E$.

If $y \in V_{0}$ is arbitrary then $\varphi^{-1}(y) \subset U$, so that every $x \in \varphi^{-1}(y)$ is contained in an $U\left(x_{j}\right)$ for some $1 \leqq j \leqq n$. By construction of $U\left(x_{j}\right)$ we have $\psi(x) \subset V_{2}\left(x_{j}\right)$. But $y \in V_{0} \subset V_{1}\left(x_{j}\right)$ and $V_{1}\left(x_{j}\right) \times V_{2}\left(x_{j}\right) \subset T \times T \backslash G$. Hence $M(y) \cap \psi(x)=\varnothing$ and $V_{0} \subset T \backslash E$.

LEMMA 4.3. If $\varphi, \psi: X \rightarrow T$ are coincidence free and if $\varphi$ is open and $\psi$ is either continuous or usc and connected-valued, then there exists for every $y \in E$ a point $z \in E$ such that $y<z$.

Proof. (i) We first assume that $\psi$ is continuous. As $\varphi$ and $\psi$ are coincidence free there exists for every $y \in E$ an $x \in \Phi^{-1}(y)$ such that $[M(y) \backslash\{y\}] \cap \psi(x) \neq \varnothing$. Choose a $w \in[M(y) \backslash\{y\}] \cap \psi(x)$, then $y<w$ and we can find a $t$ such that $y<t<w$. As $\psi$ is lsc there exists an open $U$ containing $x$ such that $[M(t) \backslash\{t\}] \cap \psi\left(x^{\prime}\right) \neq \varnothing$ for all $x^{\prime} \in U$. But $\varphi$ is open and hence $\varphi(U)=V$ is an open set containing $y$. It follows from Lemma 2.4 that there exists a $z \in V$ with $y<z<t$. Choose $x^{\prime} \in U$ such that $z \in \varphi\left(x^{\prime}\right)$. Then $[M(t) \backslash\{t\}] \cap \psi\left(x^{\prime}\right) \neq \varnothing$ implies $\psi\left(x^{\prime}\right) \cap M(z) \neq \varnothing$, hence $z \in E$.

(ii) The proof can now easily be adjusted to the case where $\psi$ is usc and connected-valued if we keep in mind that Lemma 2.3 implies 
here that if $[M(y) \backslash\{y\}] \cap \psi(x) \neq \varnothing$ then $\psi(x) \subset M(y) \backslash\{y\}$. We define $w$ as the zero of $\psi(x)$ which exists according to Lemma 2.2, and determine an open set $U$ containing $x$ so that $\psi(U) \subset M(t) \backslash\{t\}$, which is possible as $\psi$ is usc. The rest is completely analogous.

Proof of Theorem 4.1. As $\varphi$ is onto the set $E$ contains the zero of $T$ and hence is nonempty. If $\varphi$ and $\psi$ have no coincidence then it follows from Lemmas 4.2 and 4.3 that $E$ is closed but has no maximal element. But this contradicts Lemma 2.1.

We now consider the case where $\varphi$ is monotone instead of open.

THEOREM 4.4. If $\varphi: X \rightarrow T$ is usc and monotone and $\psi: X \rightarrow T$ is either continuous or usc and connected-valued then $\varphi$ and $\psi$ have a coincidence.

Proof. The theorem can be proved in the same way as Theorem 4.1 by studying the set $E$. Hence it is only necessary to prove the counterpart of Lemma 4.3, which we shall do now.

LEMma 4.5. If $\varphi, \psi: X \rightarrow T$ are coincidence free and if $\varphi$ is usc and monotone and $\psi$ is either continuous or usc and connected-valued, then there exists for every $y \in E$ a point $z \in E$ such that $y<z$.

Proof. (i) Again we assume first that $\psi$ is continuous. Take any $y \in E$ and let $B=\varphi^{-1}(y)$. As $\varphi$ is usc and monotone $B$ is closed and connected. Further $y \notin \psi(B)$ as $\varphi$ and $\psi$ are coincidence free. As $y \in E$ we have $[M(y) \backslash\{y\}] \cap \psi(B) \neq \phi$. Choose $v \in[M(y) \backslash\{y\}] \cap \psi(B)$, then $y<v$. As $\psi$ is usc $\psi(B)$ is closed, hence if $m$ is the maximum of $[y, v] \cap \psi(B)$ then $y<m$. Choose $t$ such that $y<t<m$. We next show that $[M(t) \backslash\{t\}] \cap \psi(x) \neq \varnothing$ for every $x \in B$. Define

$$
B^{\prime}=\{x \in B \mid[M(t) \backslash\{t\}] \cap \psi(x) \neq \varnothing\} .
$$

As $\psi$ is lsc there exists for every $x \in B^{\prime}$ an open set $U^{\prime}$ containing $x$ such that $[M(t) \backslash\{t\}] \cap \psi\left(x^{\prime}\right) \neq \varnothing$ for every $x^{\prime} \in U^{\prime}$. Hence $U^{\prime} \cap B \leqq B^{\prime}$, i.e. $B^{\prime}$ is open in $B$. As $\psi$ is use there exists for every $x \in B \backslash B^{\prime}$ an open set $U^{\prime \prime}$ containing $x$ such that $\psi\left(U^{\prime \prime}\right) \subset T \backslash M(t)$. Hence $U^{\prime \prime} \cap B \subseteq B \backslash B^{\prime}$, i.e. $B \backslash B^{\prime}$ is open in $B$. Therefore $B^{\prime}$ is both open and closed in the connected set $B$, and as $B^{\prime} \neq \varnothing$ it follows that $B^{\prime}=B$.

Hence if $x$ is an arbitrary point of $B$ then $[M(t) \backslash\{t\}] \cap \psi(x) \neq \varnothing$. As $\psi$ is lsc there exists an open set $U(x)$ containing $x$ such that $[M(t) \backslash\{t\}] \cap \psi\left(x^{\prime}\right) \neq \varnothing$ for every $x^{\prime} \in U(x)$. Define $U=U\{U(x) \mid x \in B\}$, 
then $U$ is an open set containing $B$. Now choose $w$ such that $y<w<t$ and let $A=\varphi^{-1}[y, w]$. Lemma 3.2 shows that $A$ is a closed and connected set, and it contains $B$ as a proper subset.

If $U \cap(A \backslash B)=\varnothing$, then $B=U \cap B=(U \cap B) \cup(U \cap(A \backslash B))=$ $U \cap A$ is open in $A$. But it is also closed in $A$, and $A$ is connected. As this is impossible we have $U \cap(A \backslash B) \neq \varnothing$. Now take any $x \in U \cap(A \backslash B)$. Then $\varphi(x) \cap([y, w] \backslash\{y\}) \neq \varnothing$, and we can choose a $z \in \varphi(x)$ such that $y<z \leqq w$. As $x \in U$ we have $[M(t) \backslash\{t\}] \cap \psi(x) \neq \varnothing$ and hence $M(z) \cap \psi(x) \neq \varnothing$. Therefore $z$ is in $E$ and $y<z$.

(ii) We now adjust the proof to the case where $\psi$ is usc and connected-valued, using again that here Lemma 2.3 shows that $\psi(x) \subset M(t) \backslash\{t\}$ if $[M(t) \backslash\{t\}] \cap \psi(x) \neq \varnothing$, and that Lemma 2.2 shows that $\psi(x)$ has a zero. For any $y \in E$ we choose an $x \in \varphi^{-1}(y)$ with $\psi(x) \subset M(y) \backslash\{y\}$ and take $v$ as the zero of $\psi(x)$. In order to show that $B^{\prime}$ is open in $B$ we can use the fact that if $x \in B^{\prime}$ then $\psi(x) \subset M(t) \backslash\{t\}$. As $\psi$ is usc there exists then an open set $U^{\prime}$ containing $x$ such that $\psi\left(U^{\prime}\right) \subset M(t) \backslash\{t\}$, so that $U^{\prime} \cap B \subseteq B^{\prime}$. The argument that $B^{\prime}$ is closed in $B$ needs no change. In the construction of the open cover $U=$ $\cup\{U(x) \mid x \in B\}$ of $B$ we use the fact that $\psi$ is usc and connected-valued to choose the open set $U(x)$ containing $x$ such that $\psi(U(x)) \subset M(t) \backslash\{t\}$. The rest does not need any modification.

Following [4] we say that the function $\varphi: X \rightarrow T$ is coincidence producing for all functions $\psi: X \rightarrow T$ if every pair $\varphi, \psi$ has a coincidence. Using this terminology we can sum up the results of this paragraph as follows.

THEOREM 4.6. Any usc function $\varphi: X \rightarrow T$ from a compact Hausdorff space $X$ onto a tree $T$ is coincidence producing for all functions $\psi: X \rightarrow T$ which are either continuous or usc and connected-valued if it is either open or monotone.

5. Applications to fixed points. A fixed point of a multifunction $\varphi: X \rightarrow X$ is a point $x$ such that $x \in \varphi(x)$. The close relation between the existence of fixed points and coincidences is shown in the following lemma.

Lemma 5.1. Let $\varphi_{1}: Y \rightarrow X$ and $\varphi_{2}: X \rightarrow Y$ be multifunctions. Then the composite function $\varphi_{2} \circ \varphi_{1}: Y \rightarrow Y$ has a fixed point if and only if the two functions $\varphi_{1}^{-1}: \varphi_{1}(Y) \rightarrow Y$ and $\varphi_{2} \mid \varphi_{1}(Y): \varphi_{1}(Y) \rightarrow Y$ have a coincidence.

The proof is immediate from the definitions of fixed points and coincidences. 
THEOREM 5.2. The product $\varphi=\varphi_{2} \circ \varphi_{1}: T \rightarrow T$ of a $\left\{\begin{array}{l}\text { continuous } \\ \text { connected- }\end{array}\right.$ valued and usc $\}$ function $\varphi_{1}: T \rightarrow X$ from a tree $T$ into a compact Hausdorff space $X$ and a $\begin{aligned} & \text { connected-valued and usc } \\ & \text { continuous }\end{aligned}$ function $\varphi_{2}: X \rightarrow T$ has a fixed point.

Proof. $\varphi_{1}(T)$ is a compact Hausdorff subset of $X$ as $\varphi_{1}$ is usc. Hence it follows from Lemma 3.1 and $\left(\varphi^{-1}\right)^{-1}=\varphi$ that $\varphi_{1}^{-1}: \varphi_{1}(T) \rightarrow T$ is usc and open if and only if $\varphi_{1}: T \rightarrow X$ is continuous, and that $\varphi_{1}^{-1}: \varphi_{1}(T) \rightarrow T$ is usc and monotone if and only if $\varphi_{1}: T \rightarrow X$ is usc and connected-valued. Therefore the theorem is a consequence of Lemma 5.1 and the coincidence Theorem 4.1 and 4.4.

With $X=T$ and either $\varphi_{2}=$ identity map or $\varphi_{1}=$ identity map we obtain from Theorem 5.2.

Corollary 5.3. Any continuous or usc and connected-valued function of a tree into itself has a fixed point.

The case of continuous functions was proved for dendrites (i.e. metric trees) by Plunkett [3] and extended to topologically chained, hereditarily unicoherent and hereditarily decomposable continua by Ward [8]. The case of usc and connected-valued functions is the fixed point theorem of Wallace [5] and Capel and Strother [2]. We see how Theorem 5.2 composes these two cases.

\section{REFERENCES}

1. C. Berge, Topological spaces, Edinburgh and London, 1963.

2. C. E. Capel and W. L. Strother, Multi-valued functions and partial order, Portugal. Math. 17 (1958), 41-47.

3. R. L. Plunkett, A fixed point theorem for continuous multivalued transformations, Proc. Amer. Math. Soc. 7 (1956) 160-163.

4. H. Schirmer, Coincidence producing maps onto trees, Canad. Math. Bull. 10 (1967), 417-423.

5. A. D. Wallace, A fixed point theorem for trees, Bull. Amer. Math. Soc. 47 (1941), 757-760.

6. L. E. Ward, Jr., Partially ordered topological spaces, Proc. Amer. Math. Soc. 5 (1954), 144-161.

7. — A note on dendrites and trees, Proc. Amer. Math. Soc. 5 (1954), 992-994.

8. _ 921-927.

9. - A general fixed point theorem, Colloq. Math. 15 (1966), 243-251. 
10. G. T. Whyburn, Continuity of multifunctions, Proc. Nat. Acad. Sci. U. S. A. 54 (1965), 1494-1501.

Received November 21, 1969, and in revised form March 25, 1970. This research was partially supported by the National Research Council of Canada (Grant A 7579).

Carleton University

OtTawa, Canada 



\section{PACIFIC JOURNAL OF MATHEMATICS}

\section{EDITORS}

H. SAMELSON

Stanford University

Stanford, California 94305

\section{Richard Pierce}

University of Washington

Seattle, Washington 98105
J. DugundJI

Department of Mathematics

University of Southern California

Los Angeles, California 90007

RICHARD ARENS

University of California

Los Angeles, California 90024

\section{ASSOCIATE EDITORS}

\section{E. F. BeCKenBACH}

B. H. NeUmanN
K. YosHida

\section{SUPPORTING INSTITUTIONS}

\author{
UNIVERSITY OF BRITISH COLUMBIA \\ CALIFORNIA INSTITUTE OF TECHNOLOGY \\ UNIVERSITY OF CALIFORNIA \\ MONTANA STATE UNIVERSITY \\ UNIVERSITY OF NEVADA \\ NEW MEXICO STATE UNIVERSITY \\ OREGON STATE UNIVERSITY \\ UNIVERSITY OF OREGON \\ OSAKA UNIVERSITY \\ UNIVERSITY OF SOUTHERN CALIFORNIA
}

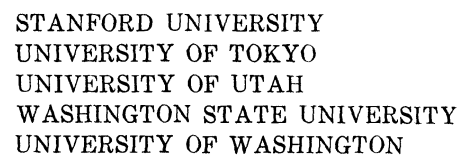

STANFORD UNIVERSITY UNIVERSITY OF TOKYO

UNIVERSITY OF UTAH

WASHINGTON STATE UNIVERSITY

UNIVERSITY OF WASHINGTON

The Supporting Institutions listed above contribute to the cost of publication of this Journal, but they are not owners or publishers and have no responsibility for its content or policies.

Mathematical papers intended for publication in the Pacific Journal of Mathematics should be in typed form or offset-reproduced, (not dittoed), double spaced with large margins. Underline Greek letters in red, German in green, and script in blue. The first paragraph or two must be capable of being used separately as a synopsis of the entire paper. The editorial "we" must not be used in the synopsis, and items of the bibliography should not be cited there unless absolutely necessary, in which case they must be identified by author and Journal, rather than by item number. Manuscripts, in duplicate if possible, may be sent to any one of the four editors. Please classify according to the scheme of Math. Rev. Index to Vol. 39. All other communications to the editors should be addressed to the managing editor, Richard Arens, University of California, Los Angeles, California, 90024.

50 reprints are provided free for each article; additional copies may be obtained at cost in multiples of 50 .

The Pacific Journal of Mathematics is published monthly. Effective with Volume 16 the price per volume (3 numbers) is $\$ 8.00$; single issues, $\$ 3.00$. Special price for current issues to individual faculty members of supporting institutions and to individual members of the American Mathematical Society: $\$ 4.00$ per volume; single issues $\$ 1.50$. Back numbers are available.

Subscriptions, orders for back numbers, and changes of address should be sent to Pacific Journal of Mathematics, 103 Highland Boulevard, Berkeley, California, 94708.

PUBLISHED BY PACIFIC JOURNAL OF MATHEMATICS, A NON-PROFIT CORPORATION

Printed at Kokusai Bunken Insatsusha (International Academic Printing Co., Ltd.), 7-17, Fujimi 2-chome, Chiyoda-ku, Tokyo, Japan. 


\section{Pacific Journal of Mathematics}

\section{Vol. 34, No. 3 \\ July, 1970}

Richard Hindman Bouldin, The peturbation of the singular spectrum

Hugh D. Brunk and Søren Glud Johansen, A generalized Radon-Nikodym derivative .

Henry Werner Davis, F. J. Murray and J. K. Weber, Families of $L_{p}$-spaces

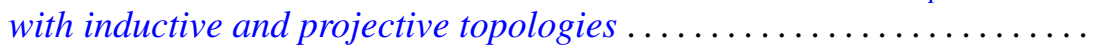

Esmond Ernest Devun, Special semigroups on the two-cell .

Murray Eisenberg and James Howard Hedlund, Expansive automorphisms

of Banach spaces ......................................

Frances F. Gulick, Actions of functions in Banach algebras.

Douglas Harris, Regular-closed spaces and proximities.

Norman Lloyd Johnson, Derivable semi-translation planes . .

Donald E. Knuth, Permutations, matrices, and generalized Young

tableaux..........................................

Herbert Frederick Kreimer, Jr., On the Galois theory of separable

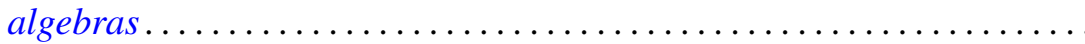

You-Feng Lin and David Alon Rose, Ascoli's theorem for spaces of

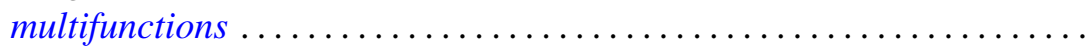

David London, Rearrangement inequalities involving convex functions . . . .

Louis Pigno, A multiplier theorem.

749

Helga Schirmer, Coincidences and fixed points of multifunctions into trees.

755

Richard A. Scoville, Some measure algebras on the integers .

Ralph Edwin Showalter, Local regularity of solutions of Sobolev-Galpern

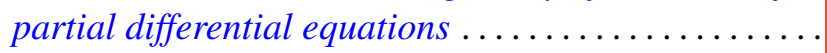

Allan John Sieradski, Twisted self-homotopy equivalences

John H. Smith, On S-units almost generated by S-units of subfields ...

803

Masamichi Takesaki, Algebraic equivalence of locally normal

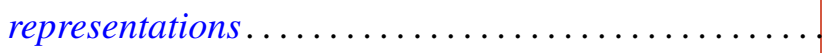

Joseph Earl Valentine, An analogue of Ptolemy's theorem and its converse in

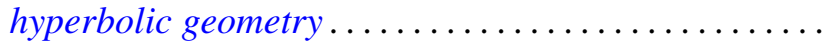

David Lawrence Winter, Solvability of certain p-solvable linear groups of finite order 